\title{
From conventional drug therapy to a broader systemic view: openness towards new and revised landmarks
}

\author{
J. Buxeraud ${ }^{1}$, L. Sergheraert ${ }^{2}$ \\ ${ }^{1}$ Department of Medicinal Chemistry, Faculty of Pharmacy, University of Limoges, Limoges, France \\ ${ }^{2}$ Santaure Conseil, Dooloo, France
}

The gastrointestinal tract is the most frequently used way of access for any type of oral therapies and a preferential one for Medical Devices Made of Substances. The socalled complex diseases, which represent a huge part of medical activity, generally result from the complex interaction between biological, psychological, genetic and environmental factors. In fact, approaching the disease from the perspective of the symptom or dysfunction of a single receptor does not always make it possible to effectively manage the patient in all her/his complexity. A systemic approach that considers the whole patient on a totality, capable of regulating her/himself to maintain a homeostatic balance allows to act both on the symptoms and on the signals resulting from all of the organism's interactions, without seeking to identify cause-and-effect relationships. The Medical Devices Made of Substances (MDMS), whose physiological non-pharmacological mechanisms of action allow to regulate the homeostatic balance of the organism, seem particularly useful to complement our therapeutic arsenal. Our knowledge in physiology combined with our better understanding of the pathophysiological mechanisms of the main functions of the organism should encourage us, pharmacologists, doctors and competent health authorities, to take another look both on the way to apprehend medicine and on the evaluation of therapeutic products and their regulatory requirements to access the market.

\section{Towards a paradigm shift in care}

Medicine is undergoing profound changes. Acute diseases are gradually supplanted by chronic diseases, themselves generally induced by the lifestyle inherent in our modern society and the growing ageing of the population. Infectious diseases, which, for a long time, have been a major cause of morbidity and mortality, are being better and better controlled.

On the other hand, there appeared, in greater number, socalled complex diseases which now represent a huge part of medical activity, with multifactorial origins, at the same time biological, psychological, genetic and environmental and mechanisms of occurrence which are still little known (1). Typically, this is the case with type 2 diabetes, high blood pressure, congestive heart failure or even digestive problems, whose pharmacological treatments have become considerably more complex over the 
Buxeraud J., Sergheraert L.

past ten years. It is also not uncommon for patients to have multiple complex illnesses simultaneously, which further complicates the disease management. Approaching the disease from the perspective of the main receptor dysfunction based on the targeted symptom does not always make it possible to effectively manage the patient in all its complexity. This approach is reductive and explains the current difficulties to an efficient care management. A systemic approach to the patient that takes into account the dynamic interactions between the impaired functions and their effects rather than the individual receptors correlated to the symptoms appears fundamental to both control the symptoms and rebalance the physiological processes, without attempting the causeand-effect relationships. This approach, which overturns the traditional landmarks of medicine, acts both on the symptoms and also on the signals resulting from all of the organism's interactions in a system of complex, multiple and circular causalities. In the gastrointestinal system and more specifically in Irritable Bowel Syndrome, an extra-digestive complaint may be the cause and / or the consequence of the disorders. Indeed, central signals such as stress modulate motor skills and digestive permeability. Conversely, digestive events such as fermentation of food in the colon stimulate certain structures of the digestive barrier and activate nervous networks or digestive secretions which are all signals transmitted to the central nervous system.

Thus, a more suitable approach, such as the strengthening of the digestive epithelial barrier, is more and more preferred and shows the limits of the approach targeted on a receptor (2,3). Karl Ludwig von Bertalanffy (19011972) has already criticized the reductionism of the natural sciences and said that "living organisms do not follow the same rules as physics" and therefore "we cannot reduce the biological, behavioral and social at the physical level": the first are open systems and the last is a closed system.

He highlighted the need to get out of mechanics to move towards relativity and complexity.

He proposed to consider the observed phenomena as "systems or sets of elements interrelated with each other and with the environment" (4).

\section{From Pharmacology to therapy: opportunities and perspectives}

The Medical Devices Made of Substances (MDMS) with a non-pharmacological mechanism of action to regulate the homeostatic balance of the organism seem particularly interesting and useful to complement our therapeutic arsenal. As Dr. Marco Racchi mentioned their "non-targeted" and non-pharmacological mechanism of action appear to be closer to a physiological mechanism of action (5). Some authors even speak of a therapeutic revolution. These complex substances scientifically validated according to the criteria of Evidence-Based Medicine cannot be assimilated to drugs because of their physiological and non-pharmacological mechanism of action. As Dr. Marco Racchi points out "In so far as the aim is the same: to correct a disorder that interferes with the healthy functioning of the body, it is time to enhance the concept of mechanism of action" (6). From a therapeutic point of view, we cannot deny the very close correlation that exists between substance-based medical devices and drugs. Moreover, their definitions overlap.

These products are likely to have the same indication (treatment or prevention of diseases) but the mechanism of action by which they achieve their own therapeutic effect is different. In the history of therapeutic, there are already drugs with a non-pharmacological, immunological or metabolic mechanism of action.

For example, drugs with an antacid effect and drugs with a laxative effect. These drugs are under the same regulation of drugs with a pharmacological mechanism of action.

This should make us reflect! On the other hand, innovative solutions with a non-pharmacological mechanism of action have not been developed under these conditions. So how can we accept today that these innovative solutions, which may have the same effect...but a different mechanism of action, are under the same pharmaceutical legislation?

In the development of a drug, we mainly focus on its molecular mechanism of action. Would it not be appropriate now to focus on the mechanism of physiological action, when the therapeutic objective is to relieve sym- 
ptoms or a functioning of our organism which has been altered? Isn't it time to change our vision of molecular pharmacology and also our vision of non-pharmacological mechanism of action?

Our knowledge in physiology combined with our better understanding of the pathophysiological mechanisms of the main functions of the organism should encourage us, pharmacologists, doctors and competent health authorities, to take another look both on the way to apprehend medicine and on the evaluation of therapeutic products and their regulatory requirements to access the market.

\section{References}

1. Klimek $P$, et al. Disentangling genetic and environmental risk factors for individual diseases from multiplex comorbidity net- works. Sci. 2016. Rep. 6, 39658; doi: 10.1038/srep39658.

2. Camilleri M, et al. Intestinal barrier function in health and gastrointestinal disease. Neurogastroenterol Motil. 2012; 24 (6): 503-512.

3. Camilleri M, et al. Irritable Bowel Syndrome: Methods, Mechanisms and Pathophysiology. The confluence of increased permeability, inflammation, and pain in irritable bowel syndrome. Am J Physiol Gastrointest Liver Physiol. 2012; 303: G775G785.

4. Drack M. Ludwig von Bertalanffy's Organismic View on the Theory of Evolution. J Exp Zool B Mol Dev Evol. 2015 Mar; 324(2): 77-90.

5. Von Bertalanffy L. The History and Status of General Systems: The Academy of Management Journal, General Systems Theory. $1972 ; 15$ (4): 407-426.

6. Racchi M, Govoni S. The concept of non-pharmacological mechanism of action in medical devices made of substances in practice: what pharmacology can do to promote the scientific implementation of the European medical device regulation. Pharmadvances, in press. 